\title{
ON THE CONVERGENCE OF SEQUENCES OF OPERATORS AND THE CONVERGENCE OF THE SEQUENCE OF THEIR SPECTRA
}

\author{
SEN-ZHONG HUANG, HERMANN RENDER and MANFRED P. H. WOLFF
}

\begin{abstract}
Let $\left(T_{n}\right)$ be a sequence of bounded linear invertible operators on the Banach space $E$ over $\mathrm{C}$, and assume that the double sequence $\left(T_{n}^{k}\right)_{n \in \mathrm{N}, k \in \mathrm{Z}}$ satisfies a certain growth condition. We prove the following theorem:

If the sequence $\left(\sigma\left(T_{n}\right)\right)_{n \in \mathrm{N}}$ of the spectra $\sigma\left(T_{n}\right)$ of $T_{n}$ converges to the singleton $\{1\}$ with respect to the Hausdorff metric on bounded subsets of $\mathrm{C}$, then $\left(T_{n}\right)$ converges uniformly to the identity operator. We also establish a generalization of this result.
\end{abstract}

\section{Introduction}

In the following let $E$ be a fixed Banach space over C. Let $\left(T_{n}\right)_{n \in \mathrm{N}}$ be a sequence of bounded linear operators on $E$ which converges uniformly (i.e. with respect to the operator norm) to the identity $I$ on $E$. Then the sequence $\left(\sigma\left(T_{n}\right)\right)_{n \in \mathrm{N}}$ of the spectra $\sigma\left(T_{n}\right)$ of $T_{n}$ converges to $\{1\}$ with respect to the Hausdorff metric which is defined for closed bounded sets $A, B$ in two steps:

Set $\operatorname{dist}(A, B):=\sup _{a \in A}\left(\inf _{b \in B}|a-b|\right)$, then

$$
d(A, B):=\max (\operatorname{dist}(\mathrm{A}, \mathrm{B}), \operatorname{dist}(\mathrm{B}, \mathrm{A})) .
$$

It is the main aim of our paper to prove a certain converse to this result. To this end let us first of all consider the "stationary" case, i.e. let $T$ be a bounded linear operator with $\sigma(T)=\{1\}$. Then in general $T \neq I$. But a very old and famous theorem of Gelfand [4] says that if in addition $\left(T^{k}\right)_{k \in Z}$ is norm bounded then $T=I$.

This theorem was generalized by many authors; we cite some of them: E. Hille [7] proved the result under the condition that $\left(\left\|T^{k}\right\|\right)=o(k)$. If one applies a result of Shilov $[5, \S 41]$ together with the representation theory of

Research for this paper was carried out at the Workshop/Symposium NSA 96 at the ICMS Edinburgh, with support from the UK EPSKRC/ICMS which is gratefully acknowledged by the third author (M.P.H.W.)

Received January 17, 1996. 
Domar and Lindahl [3] then one gets the result under the conditions $\left(\left\|T^{k}\right\|\right)=0\left(|k|^{\alpha}\right)$ for some $\alpha \geq 0$ together with $\liminf _{k \rightarrow \pm \infty} \frac{\left\|T^{k}\right\|}{k}=0$. Atzmon [1] showed that the conditions (1) $\sup \left\{\left\|T^{k}\right\|: k \geq 0\right\}<\infty$ and $\lim _{k \rightarrow \infty} \frac{\log \left\|T^{-k}\right\|}{\sqrt{k}}=0$ together imply $T=I$. Other generalizations are to be found in [14] and [10]. The up to now most general case is treated in [9, §3]. We shall show that if the sequence $\left(T_{n}\right)$ satisfies uniformly one of the conditions mentioned above and if $\left(\sigma\left(T_{n}\right)\right)$ converges to $\{1\}$ then $\left(T_{n}\right)$ converges uniformly to $I$.

Our paper is organized in the following manner: In the second section we recall some important facts on the Beurling algebras $l_{w}^{1}(\mathbf{Z})$ which we need in the sequel, in the third section we state and prove our main theorem where as the fourth section is devoted to a generalization of it.

\section{Preliminaries}

We start by a recapitulation of what we need about the Beurling algebra $l_{w}^{1}(Z)$ for a given weight $w$ on $\mathbf{Z}$ (for notions not explained here we refer to [11]).

Definition 2.1. (a) A function $w: Z \rightarrow[1, \infty[=\{x \in \mathbf{R}: x \geq 1\}$ is called a weight if $w(k+l) \leq w(k) w(l)$ holds for all $k, l$.

(b) Such a weight is called nonquasianalytic (nqa for short) if

$$
\sum_{k \in Z} \frac{\log w(k)}{1+k^{2}}<\infty
$$

For $w$ being a weight the space $\left\{f \in \mathrm{C}^{\mathbf{Z}}: \sum_{k \in \mathbf{Z}}|f(k)| w(k)<\infty\right\}=: l_{w}^{1}(\mathbf{Z})$ is a subalgebra of $l^{1}(\mathbf{Z})$ with respect to convolution, which is a Banach algebra when equipped with the norm

$$
\|f\|=\sum_{k \in \mathbf{Z}}|f(k)| w(k) .
$$

If $w$ is nonquasianalytic then it follows from the theory of Domar [2] on general Beurling algebras that $l_{w}^{1}(\mathbf{Z})$ is a Wiener algebra with an approximate unit (see [11] for these notions).

Let $\Gamma$ be the Gelfand space of $l_{w}^{1}(\mathbf{Z})$. If $w$ is nonquasianalytic then $\Gamma$ can be identified with $\mathbf{T}:=\{\xi \in \mathbf{C}:|\xi|=1\}$ by setting $\varphi_{\xi}(f)=\sum f(k) \xi^{k}=: \widehat{f}(\xi)$.

If $J$ is an ideal of $\mathscr{A}=l_{w}^{1}(\mathbf{Z})$ then $h(J)=\{\xi \in \Gamma: \hat{f}(\xi)=0$ for all $f \in J\}$ is called the hull of $J$. It is always a closed subset of $\Gamma$. If conversely $\Delta \subset \Gamma$ is a closed set then we set $k(\Delta)=\{f \in \mathscr{A}: \widehat{f}(\Delta)=\{0\}\}$ and $m(\Delta)=$ 
$\overline{\{f \in \mathscr{A}: f \text { vanishes on an open set containing } \Delta\}}, m(\Delta)$ is the smallest closed ideal $J$ satisfying $h(J)=\Delta$, and $k(\Delta)$ is the largest one.

Definition 2.2 (cf. [9, Definition 3.3]). The $n q a$-weight $w$ is called a weight of uniqueness ( $U$-weight for short) if $m(\{\xi\})=k(\{\xi\})$ for every $\xi \in \Gamma$.

REMARK 2.3. A closed ideal $J$ is called primary if the hull $h(J)$ is a singleton. $w$ is an $U$-weight if every primary ideal is maximal.

Concerning concrete examples we mention the following ones:

THEOREM 2.4. (a) Let $(w(k))=0\left(|k|^{\alpha}\right)$ for some $\alpha \geq 0$ and in addition let $\liminf _{k \rightarrow \pm \infty} \frac{w(k)}{k}=0$. Then $w$ is an $U$-weight.

(b) Assume that

$$
\lim _{n \rightarrow \infty}\left(\frac{w(n)}{n^{\alpha}}+\frac{\log (n)}{\sqrt{n}}\right)=0 \text { for some } \alpha \geq 0 .
$$

If $0 \leq \alpha<1$ or $\liminf _{n \rightarrow \pm \infty} w(n)<\infty$ then $w$ is an $U$-weight.

REMark 2.5. Part (a) is Shilov's theorem mentioned in the introduction. The corresponding assertion for $\alpha<1$ in part (b) was announced in [6, Theorem 8.1 (i)], and a complete proof of it was given in [1]. The other assertion of part (b) is due to the first author [9, Prop. 3.12].

We shall make heavy use of results of Domar and Lindal [3] specialized to our situation of representations of $l_{w}^{1}(\mathrm{Z})$.

Let $T$ be a bounded linear invertible operator on the Banach space $E$. Then $w(k):=\max \left(1,\left\|T^{k}\right\|\right)$ defines a weight. Let $U f:=\sum f(k) T^{k}$ for $f \in \mathscr{A}:=l_{w}^{1}(\mathrm{Z})$. Then $U$ is a contractive representation of $\mathscr{A}$ in $L(E)$. We denote by $\operatorname{ker}(U)$ its kernel $U^{-1}(\{0\})$.

Theorem 2.6. (cf. [3, Theorem 6.7], [13, Prop. 3.6 ], [8, Prop. 1.3.8]) Let w be nonquasianalytic. Then $h(\operatorname{ker}(U))=\sigma(T)$ where $\sigma(T)$ denotes the spectrum of $T$.

This theorem gives us back our results in the first section concerning the " $T=I$ " problem.

Corollary 2.7 (cf. [9, Theorem 3.10]). Let $w$ be an U-weight. Then $\sigma(T)=\{1\}$ implies $T=I$.

Proof. Since $w$ is an $U$-weight and $\sigma(T)=\{1\}$, the kernel of $U$ has codimension 1. It follows that $\mathscr{A} / \operatorname{ker}(U)=\mathrm{C} 1$ and the assertion follows from [13], Remark 3.7 (2) to Proposition 3.6. 


\section{The main result}

THEOREM 3.1. Let $\left(T_{n}\right)_{n \in \mathrm{N}}$ be a sequence of bounded linear invertible operators on the Banach space E. Assume that there exists an $U$-weight $w$ on $\mathbf{Z}$ such that $\left\|T_{n}^{k}\right\| \leq w(k)$ for all $n \in \mathrm{N}$ and $k \in \mathbf{Z}$. If the sequence $\left(\sigma\left(T_{n}\right)\right)$ of the spectra $\sigma\left(T_{n}\right)$ of $T_{n}$ converges to $\{1\}$ (with respect to the Hausdorff metric) then $\left(T_{n}\right)$ converges uniformly to the identity.

In order to prove this theorem and related results we use the theory of ultraproducts. (Compare the following paragraph with the introduction of section 2 of [12])

Let $\mathscr{U} \subset \mathscr{P}(\mathrm{N})$ be a free ultrafilter and denote by $m_{\mathscr{U}}$ the finitely additive $\{0,1\}$-valued measure on $\mathrm{N}$, given by $m_{\mathscr{U}}(A)=1$ iff $A \in \mathscr{U}$. Note that for such a measure the intersection of finitely many sets of measure 1 has also measure 1 .

Denote by $E_{\infty}$ the Banach space of all bounded sequences $\tilde{x}=\left(x_{n}\right)_{n \in \mathrm{N}}$ with $x_{n} \in E$ for all $n$, equipped with the norm $\|\tilde{x}\|=\sup _{n}\left\|x_{n}\right\|$. Then $E_{0}:=\left\{\tilde{x}: \lim _{\mathscr{U}}\left\|x_{n}\right\|=0\right\}$ is a closed subspace of $E_{\infty}$. The quotient $\widehat{E}:=E_{\infty} / E_{0}$ is called the ultrapower of $E$ with respect to $\mathscr{U}$. The norm on $\widehat{E}$ is given by $\|\widehat{y}\|=\left\|\tilde{y}+E_{0}\right\|=\lim _{\mathscr{U}}\left\|y_{n}\right\| . E$ is isometrically embedded into $\widehat{E}$ by means of $x \rightarrow(x, x, x, \ldots)+E_{0}$.

Every bounded sequence $\left(S_{n}\right)$ of operators $S_{n}$ on $E$ defines an operator $\tilde{S}$ on $E_{\infty}$ by $\tilde{S} \tilde{x}=\left(S_{n} x_{n}\right)_{n \in \mathrm{N}}$, with norm given by $\|\tilde{S}\|=\sup _{n}\left\|S_{n}\right\|$. So $E_{0}$ is invariant for $\tilde{S}$, and we obtain a uniquely defined operator $\widehat{S}$ on $\widehat{E}$ given by $\widehat{S} \hat{y}=\tilde{S} \tilde{y}+E_{0}$. Moreover, $\|\widehat{S}\|=\lim _{\mathscr{U}}\left\|S_{n}\right\|$. If $\left(R_{n}\right)$ is another bounded sequence of operators $R_{n}$ on $E_{n}$ such that $R_{n}=S_{n} m_{\mathscr{U}}$-a.e., then $\widehat{R}=\widehat{S}$. Thus each subfamily $\left(S_{n}\right)_{n \in M}$ with $\mu_{\mathscr{U}}(M)=1$ defines in a canonical way an operator on $\widehat{E}$ which coincides with $\widehat{S}$. It should cause no confusion if we denote the operator on $\widehat{E}$ induced by $\left(S_{n}\right)_{n \in M}$ also by $\widehat{S}=\left(\widehat{\left.S_{n}\right)}\right.$. If $T$ is a bounded operator on $E$ then the constant sequence $(T, T, \ldots)$ defines the extension $\widehat{T}$ of $T$ on $\widehat{E}$. The mapping $T \rightarrow \widehat{T}$ is an isometric embedding of the algebra $\mathscr{L}(E)$ into $\mathscr{L}(\widehat{E})$.

It will become clear from the context whether $\widehat{S}$ denotes an operator coming from a constant sequence or from an arbitrary sequence. But one should keep in mind the following two different notations: For $\left(S_{n}\right)_{n \in \mathrm{N}}$ being a bounded sequence of operators we consider not only $\widehat{S}=\left(\widehat{\left.S_{n}\right)}\right.$ but also each individual operator $\widehat{S}_{n}$, defined through the constant sequence $\left(S_{n}, S_{n}, S_{n}, \ldots\right)$.

Proposition 3.2. Let $w$ be a nonquasianalytic weight on $\mathrm{Z}$. Let $\left(T_{n}\right)$ be a sequence of bounded linear invertible operators on E satisfying $\left\|T_{n}^{k}\right\| \leq w(k)$ 
for all $n \in \mathrm{N}$ and $k \in \mathrm{Z}$. Let finally $A \subset \mathrm{T}$ be closed. Then $\lim _{n \rightarrow \infty} d\left(\sigma\left(T_{n}\right), A\right)=0$ implies $\sigma(\widehat{T})=A$.

Proof. (I) The assumption $\left\|T_{n}^{k}\right\| \leq w(k)$ for all $k$ and $n$ implies $\left\|\widehat{T}^{k}\right\| \leq w(k)$. Consider the representation $U$ of $\mathscr{A}:=l_{w}^{1}(\mathbf{Z})$ in $\mathscr{L}(\widehat{E})$ given by $U f:=\sum f(k) \widehat{T}^{k}$. Then Theorem 2.6 implies that $\sigma(\widehat{T})=h(\operatorname{ker}(U))$.

(II) For fixed $n \in \mathrm{N}$ we also consider $U_{n}: f \in \mathscr{A} \rightarrow U_{n} f:=\sum f(k) \widehat{T}_{n}^{k}$. Then Theorem 2.6 combined with [13, Theorem 3.4] yields that $\sigma\left(T_{n}\right)=\sigma\left(\widehat{T}_{n}\right)=h\left(\operatorname{ker}\left(U_{n}\right)\right)$.

(III) Now let $\lambda \in \mathrm{T} \backslash A$ be given and set $\varepsilon:=\operatorname{dist}(\lambda, \mathrm{A}) / 2$. Then there exists $n_{0}$ such that for $n \geq n_{0}$ the spectrum $\sigma\left(T_{n}\right)$ is contained in $C:=\{\xi: \operatorname{dist}(\xi, \mathrm{A}) \leq \varepsilon\}$

Since $m(C)$ is the smallest closed ideal $J$ in $\mathscr{A}$ with $h(J)=C$, and since $\sigma\left(T_{n}\right) \subset C$ we obtain $m(C) \subset \operatorname{ker}(U)_{n}=: J_{n}$ by (II). Set

$$
f=\lambda \delta_{0}-\delta_{1} \text { where } \delta_{k}(r)= \begin{cases}1, & r=k, \\ 0, & r \in \mathbf{Z} \backslash\{k\}\end{cases}
$$

Then $\widehat{f}(\xi)=0$ implies $\lambda=\xi$, hence $\widehat{f}$ does not vanish on $C$. Since $\mathscr{A}$ is a Wiener algebra, in particular regular, there exists $g \in \mathscr{A}$ such that $\widehat{f} \widehat{g}=1$ on $C$. This in turn implies that $\tilde{f}:=f+m(C)$ is invertible in the quotient algebra $\mathscr{A} / m(C)$. Let $\alpha:=\|g+m(C)\|$ be the quotient norm of $\tilde{g}:=g+m(C)$.

(IV) Since $m(C) \subset J_{n}, g+J_{n}$ is the inverse of $f+J_{n}$ in $\mathscr{A} / J_{n}$ and $\left\|g+J_{n}\right\| \leq \alpha$. Since $U_{n}$ is obviously contractive, $U_{n} g$ is the inverse of $\left(\lambda-T_{n}\right)$ and $\left\|U_{n} g\right\| \leq \alpha$, or in other words $\lambda \in \rho\left(T_{n}\right)\left(=\mathrm{C} \backslash \sigma\left(T_{n}\right)\right)$ and $\left\|\left(\lambda-T_{n}\right)^{-1}\right\| \leq \alpha$. This holds for all $n \geq n_{0}$ (see (III) above). But then $\lambda \in \rho(\widehat{T})$ by [12], Lemma 2.1.

(V) Let conversely $\lambda \in A$ be arbitrary. Then by assumption to every $n$ there exists $\lambda_{n} \in \sigma\left(T_{n}\right)$ such that $\lim _{n \rightarrow \infty} \lambda_{n}=\lambda$ holds. Assume now that $\lambda \in \rho(\widehat{T})$. Then by Lemma 2.1 of [12] there exists $\delta>0$ such that $\lambda \in \rho\left(T_{n}\right)$ and $\left\|\left(\lambda-T_{n}\right)^{-1}\right\|<\delta$ holds $m_{\mathscr{U}}$-a.e. To this $\delta$ there exists $n_{0} \in \mathrm{N}$ such that $\left|\lambda_{n}-\lambda\right|<1 / \delta$ for all $n \geq n_{0}$. But this implies $\left|\lambda_{n}-\lambda\right|<\frac{1}{\left\|\left(\lambda-T_{n}\right)^{-1}\right\|} m_{U^{-}}$-a.e. From the equation

$$
\left(\lambda_{n}-T_{n}\right)=\left(\lambda-T_{n}\right)\left(I-\left(\lambda-\lambda_{n}\right)\left(\lambda-T_{n}\right)^{-1}\right)
$$

we obtain that $\lambda_{n} \in \rho\left(T_{n}\right)$ holds $m_{U}-$ a.e, a contradiction.

Proof of 3.1: If the assertion fails there exists a free ultrafilter $\mathscr{U}$ such that $\lim _{\mathscr{U}}\left\|T_{n}-I\right\|>0$, or in other words, such that $\widehat{T} \neq I$ holds. But $\lim d\left(\sigma\left(T_{n}\right),\{1\}\right)=0$ implies $\sigma(\widehat{T})=\{1\}$ by Proposition 3.3. Now from $\left\|\vec{T}_{n}^{R}\right\| \leq w(k)$ for all $n \in \mathrm{N}$ and $k \in \mathrm{Z}$ we obtain $\left\|\widehat{T}^{k}\right\| \leq w(k)$ for all $k \in \mathrm{Z}$ (see part (I) of the proof of Prop. 3.3). Now $w$ is an $U$-weight by hypothesis, so $\widehat{T}=I$, a contradiction. 


\section{A generalization}

In this section we consider the convergence of $\left(\sigma\left(T_{n}\right)\right)$ to a finite set $\left\{\lambda_{1}, \ldots, \lambda_{r}\right\}=A$. For the formulation of our result we need the following definition:

Definition 4.1. A decomposition of the identity operator I on the Banach space $E$ is a finite set $\left\{P_{1}, \ldots, P_{r}\right\}$ of pairwise disjoint nonvanishing projections which add up to $I$, i.e. $\sum_{k=1}^{r} P_{k}=I$.

THEOREM 4.2. Let $w$ be an $U$-weight on $\mathrm{Z}$ and let $\left(T_{n}\right)$ be a sequence of bounded linear invertible operators such that $\left\|T_{n}^{k}\right\| \leq w(k)$ for all $n \in \mathrm{N}$ and $k \in \mathrm{Z}$. Assume that $\lim _{n \rightarrow \infty} d\left(\sigma\left(T_{n}\right), A\right)=0$ for some finite set $A=\left\{\lambda_{1}, \ldots, \lambda_{r}\right\}$. Then there exists $n_{0}$ such that to each $n \geq n_{0}$ there exists a partition $\left\{P_{1 n}, \ldots, P_{r n}\right\}$ of I satisfying $\lim _{n \rightarrow \infty}\left\|T_{n}-\sum_{k=1}^{r} \lambda_{k} P_{k, n}\right\|=0$

Remark 4.3. Note that the sequence $\left(\sum_{k=1}^{r} \lambda_{k} P_{k, n}\right)$ itself needs not converge as easy examples show.

Proof. (I) Let $\delta:=\inf \left\{\left|\lambda_{i}-\lambda_{j}\right|: i \neq j\right\} / 8$ and let $n_{0} \in \mathrm{N}$ be chosen so that $d\left(\sigma\left(T_{n}\right), A\right)<\delta$ for all $n \geq n_{0}$. Then, we have for $D_{k, n}:=\left\{\lambda \in \sigma\left(T_{n}\right)\right.$ : $\left.\left|\lambda-\lambda_{k}\right|<\delta\right\}$ that

(i) $D_{k, n} \cap D_{l, n}=\emptyset$ for $k \neq l$; and

(ii) $\sigma\left(T_{n}\right)=\bigcup_{k=1} D_{k, n}$.

(II) Let $P_{k, n}$ be the spectral projection corresponding to $D_{k, n}$, i.e.,

$$
P_{k, n}=\frac{1}{2 \pi i} \int_{\left|\xi-\lambda_{k}\right|=2 \delta}\left(\xi-T_{n}\right)^{-1} d \xi .
$$

The set $\left\{P_{k, n}: k=1, \ldots, r\right\}$ is a partition of $I$, and we want to prove that $\left(T_{n}-\sum_{k=1}^{r} \lambda_{k} P_{k, n}\right)$ converges to 0 .

(III) We use an ultrapower $\widehat{E}$ of $E$ with respect to an arbitrary free ultrafilter. Proposition 3.3 yields $\sigma(\widehat{T})=\left\{\lambda_{1}, \ldots, \lambda_{r}\right\}$. Let $Q_{j}$ be the spectral projection corresponding to $\lambda_{j}$; moreover set $F_{j}:=Q_{j}(\widehat{E})$. Then $\left.\bar{\lambda}_{j} \widehat{T}\right|_{F_{j}}=I$ by Theorem 2.6 since $\left\|\left(\bar{\lambda}_{j} \widehat{T}\right)^{k}\right\| \leq w(k)$ (cf. the proof of 3.1). Hence $\widehat{T}=\sum \lambda_{j} Q_{j}$.

(IV) Set $C_{k}:=\left\{\xi:\left|\xi-\lambda_{k}\right| \leq \delta\right\}$ and $C:=\bigcup_{k=1}^{r} C_{k}$. 
As in the proof of Proposition 3.3 we have $m(C) \subset \operatorname{ker}\left(U_{n}\right)=: J_{n}$ at least for $n \geq n_{0}$. The function $\xi \rightarrow g_{\xi}:=\xi \delta_{0}-\delta_{1}$ has the property that $\widehat{g}_{\xi}$ does not vanish on $C$, hence $\tilde{g}_{\xi}=: g_{\xi}+m(C)$ is invertible and uniformly continuous (as a function of $\xi$ ). Since $m(C) \subset J_{n}$ for $n \geq n_{0}$ and since $U_{n}: l_{w}^{1}(\mathrm{Z}) \rightarrow \mathscr{L}(E)$ $\left(U_{n} f:=\sum_{-\infty}^{\infty} f(k) T_{n}^{k}\right)$ is a contraction we obtain for the induced representation $\overline{U_{n}}: l_{w}^{1}(\mathrm{Z}) / J_{n} \rightarrow \mathscr{L}(E)$ that $\left(\xi-T_{n}\right)^{-1}=\overline{U_{n}}\left(\left(g_{\xi}+J_{n}\right)^{-1}\right)$ is uniformly equicontinuous. Thus for each $\varepsilon>0$ there exists a partition $\left\{\xi_{1}, \ldots \xi_{m}\right\}$ of the circle $C_{k}$ such that

$$
\left\|P_{k, n}-\frac{1}{2 \pi} \sum\left(\xi_{j}-T_{n}\right)^{-1}\left(\xi_{j+1}-\xi_{j}\right)\right\|<\varepsilon / 2 \text { uniformly in } n \geq n_{0} .
$$

Passing to $\widehat{E}$ we obtain $\left\|Q_{k}-\frac{1}{2 \pi} \sum\left(\xi_{j}-\widehat{T}\right)^{-1}\left(\xi_{j+1}-\xi_{j}\right)\right\| \leq \varepsilon / 2$ as well as $\left\|\widehat{P}_{k}-\frac{1}{2 \pi} \sum\left(\xi_{j}-\widehat{T}\right)^{-1}\left(\xi_{j+1}-\xi_{j}\right)\right\| \leq \varepsilon / 2$. Since $\varepsilon>0$ was arbitrary we get $Q_{k}=\stackrel{P}{*}_{k}$, hence $\left(\widehat{T}-\sum \lambda_{j} \widehat{P}_{k}\right)=0$. Since the free ultrafilter $\mathscr{U}$ was chosen arbitrarily the assertion follows.

\section{REFERENCES}

1. A. Atzmon, Operators which are annihilated by analytic functions, Acta Math. 144 (1980), 27-63.

2. Y. Domar, Harmonic analysis based on certain commutative Banach algebras, Acta Math. 96 (1956), 1- 66.

3. Y. Domar, L. A. Lindahl, Three spectral notions for representations of commutative Banach algebras, Ann. Inst. Fourier (Grenoble) 25 (1975), 1-32.

4. I. M. Gelfand, Zur Theorie der Charaktere der Abelschen topologischen Gruppen, Rec. Math., N.S. 9 [51] (1941), 49-50.

5. I. M. Gelfand, D. Raikov, G. Shilov, Commutative Normed Rings, Chelsea, Bronx, 1964.

6. V. P. Gurarii, Harmonic analysis in space with a weight, Trans. Moscow Math. Soc. 35 (1979), 21-75.

7. E. Hille, On the theory of characters of groups and semigroups in normed vector rings, Proc. Nat. Acad. Sci. 30 (1944), 58-60.

8. Sen-Zhong Huang, Spectral theory for non-quasianalytic representations of locally compact abelian groups, Thesis, Universität Tübingen, 1996.

9. Sen-Zhong Huang, Spectral synthesis, semi-amenability of Beurling algebras and applications, preprint, Tübingen 1996.

10. Vũ Quôc Phóng, Semigroups with nonquasianalytic growth, Studia Math. 104 (1993), no 3, 229-241.

11. R. Reiter, Classical Harmonic Analysis and Locally Compact Groups, Oxford Univ. Press, London, 1968.

12. F. Räbiger, M. Wolff, On the approximation of positive operators and the behaviour of the spectra of the approximants, Integral Equations Operator Theory 28 (1997), 72-86.

13. M. P. H. Wolff, Spectral theory of group representations and their nonstandard hull, Israel J. Math. 48 (1984), 205-224. 
78 SEN-ZHONG HUANG, HERMANN RENDER AND MANFRED P. H. WOLFF

14. J. Zemánek, On the Gelfand-Hille theorems, in: J. Zemánek (ed.): Functional Analysis and Operator Theory 30, Banach Center Publ. 30, Warszawa (1994), 369-385.

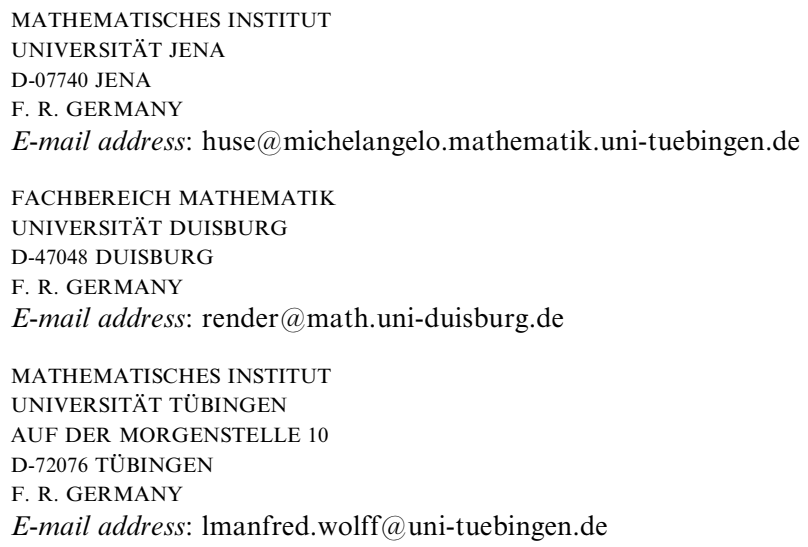

E-mail address: lmanfred.wolff@uni-tuebingen.de 